\title{
Outcomes of Complete Atrioventricular Septal Defect Surgical Repair in Down Patients
}

Ayman R. Abdelrehim ${ }^{1,2}$, Abdulhamid Alnajjar ${ }^{3}$,Syed Aitizaz FRCS ${ }^{2}$, Mostafa Ashry ${ }^{4}$, Rafik F.B. Soliman*1

${ }^{1}$ Department of Cardiothoracic Surgery, Faculty of Medicine, Menoufia University, Menoufia, Egypt. ${ }^{2}$ Department of Pediatric Cardiac Surgery, Madina Cardiac Center, Madina, Kingdom of Saudia Arabia, Khalid Bin Al Waleed Road, PO Box 6167, Post Code 41442

${ }^{3}$ Department of Pediatric Cardiology, Madina Cardiac Center, Madina, Kingdom of Saudia Arabia, Khalid Bin Al Waleed Road, PO Box 6167, Post Code 41442

${ }^{4}$ Department of Pediatric, Sohag University, Faculty of Medicine, Sohag, Egypt.

*Corresponding author: Rafik F.B. Soliman, Mobile: (+20) 01011102102, E-Mail: rafikfekry@ hotmail.com

\begin{abstract}
Background: Atrioventricular septal defect (AVSD) is commonly associated with chromosomal abnormalities, especially trisomy 21 or Down syndrome (DS). Surgical repair of complete AVSD (CAVSD) is a complex procedure that carries risks of postoperative morbidity and mortality.

Objective: To evaluate the surgical outcomes and to identify the risk factors for hospital mortality and reoperation after repair of CAVSD in DS patients.

Patients and Methods: This retrospective cohort study included 65 consecutive DS patients who underwent surgical correction for the complete form of AVSD with or without associated congenital heart diseases during the period from $1^{\text {st January }} 2014$ to the end of June 2020. Patients with associated other major cardiac anomalies were excluded. Results: In-hospital mortality was documented in $3(4.6 \%)$ patients, whereas $4(6.2 \%)$ patients needed second unplanned operation for valve/shunt correction. Heart block that needed permanent pacemaker insertion was recorded in $3(4.6 \%)$ patients. In-hospital mortality was significantly associated with prolonged cardiopulmonary bypass $(\mathrm{CPB})$ time $(\mathrm{p}=0.008)$ and the development of renal dysfunction that required dialysis or sepsis $(\mathrm{p}=0.004)$. We found a significant association between the need for second unplanned operation and type A CAVSD $(\mathrm{p}=0.041)$ and the presence of preoperative moderate/severe atrioventricular (AV) valve regurgitation as detected in the transesophageal echocardiography (TEE) $(\mathrm{p}=0.035)$.

Conclusions: In view of the incidence of the hospital mortality, reoperation, and other postoperative morbidities, we suggest that our outcomes are accepted for surgical repair of CAVSD in DS patients. The CPB time and the development of renal dysfunction that required dialysis and sepsis during the ICU care significantly contributed to the hospital mortality.
\end{abstract}

Keywords: Atrioventricular septal defect, Down syndrome, Hospital mortality, Outcomes, Reoperation.

\section{INTRODUCTION}

Atrioventricular septal defect (AVSD) is a severe congenital heart disease characterized by a spectrum of cardiac anomalies, mainly deficient atrioventricular septation and variability in atrioventricular valve morphology. The complete form of AVSD (CAVSD) includes a premium interatrial septal defect, common atrioventricular (AV) valve orifice, and an interventricular septal defectof inlet type ${ }^{(\mathbf{1})}$. The CAVSD is an uncommon malformation, and it accounts for 3\% of all congenital heart diseases ${ }^{(2)}$.

Atrioventricular septal defect arises from defective development of endocardial cushions during the prenatal period due to genetic mutations. It is commonly associated with chromosomal abnormalities, especially trisomy 21 or Down syndrome (DS) ${ }^{(3)}$. Every six patients with DS have associated AVSD. Further, Down syndrome cell adhesion molecule gene has been described to be associated with AVSD and other congenital heart diseases in these patients ${ }^{(4)}$.
Atrioventricular septal defects are very commonly diagnosed in utero or after birth by echocardiography. Three-dimensional echocardiography is very useful in the diagnosis, further delineating treatment options, and in the follow up after surgical correction ${ }^{(5,6)}$.

A diverse and challenging group of defects that require surgical correction characterizes atrioventricular septal defect. Early surgical repair of complete AVSD is the ultimate treatment option to reduce the pulmonary vascular disease ${ }^{(7)}$. Surgical repair of complete AVSD is a complex procedure that carries risks of postoperative morbidity and mortality due to various complications such as residual intracardiac shunts, atrioventricular valve regurgitation, left ventricular outflow tract obstruction, and arrhythmias ${ }^{(8)}$.

Postoperative outcomes depend on various preoperative and operative risk factors. Further, there is no conclusive evidence in the literature on the outcomes of CAVSD repair in DS patients ${ }^{(9)}$.

Therefore, this study aimed to evaluate the surgical outcomes and to identify the risk factors for 
hospital mortality and reoperation after repair of CAVSD in DS patients.

\section{PATIENTS AND METHODS}

This retrospective cohort study was conducted at the Department of Pediatric Cardiac Surgery and Pediatric Cardiology in Madina Cardiac Center (MCC) and Faculty of Medicine, Menoufia University, Egypt.

\section{Ethical approval:}

The study was conducted after the approval of the Local Research Ethics Committee of Madina Cardiac Center ,no (2021 R24). The confidentiality of the patients' data was maintained by using a coding system for every patient. Informed written consent was obtained from parents of all children participants before recruitment in the study, after explaining the objectives of the work. This work has been carried out in accordance with The Code of Ethics of the World Medical Association (Declaration of Helsinki) for studies involving humans.

\section{Eligibility criteria:}

We included consecutive Down syndrome children who underwent primary surgical repair of CAVSD. Patients with concomitant cardiovascular anomalies including Tetralogy of Fallot (TOF), coarctation of the aorta, pulmonary artery hypertension, pulmonary artery stenosis and/or previous pulmonary artery banding (PAB) were included.

Exclusion criteria: Patients had other complicated congenital cardiac anomalies were excluded from the study.

\section{Data collection:}

Data of patients presenting between the $1^{\text {st }}$ of January 2014 and the end of June 2020 were collected from the hospital patients' records. Preoperative data included sex, age and weight at the operation time, history of pulmonary artery banding, and the pressure gradient around the band as well as the time between banding and the surgical CAVSD closure. The type of CAVSD, other concomitant congenital cardiac anomalies, the anatomy of the valves as detected in the preoperative transesophageal echocardiography (TEE), and history of preoperative catheterization were recorded. Operative data included the technique of surgical CAVSD closure either single or double patch repair, the type of thymectomy, cross clamp and cardiopulmonary bypass $(\mathrm{CPB})$ times, and the intraoperative TEE findings. Data during the period of intensive care unit (ICU) admission comprising the length of stay, the duration of inotropes and mechanical ventilation, the presence of heart block and the need for permanent pacing, and early postoperative complications and/or organ failure were recorded. The long-term outcomes including in-hospital mortality (defined as death within 30 days after CAVSD repair or before hospital discharge), the need for unplanned second operation for valves correction (interventional cardiac catheterizations and permanent pacemaker placements performed during the follow up period were not included as reoperations), heart block that needed permanent pacemaker (PPM) insertion, and recurrent hospital admission were also documented.

\section{Surgical procedures:}

For balanced complete AVSD, early primary repair either with single patch closure or with two patches closure with valve annuloplasty is necessary. Two patches closure is preferred as one patch closure is associated with an increased rate of recurrent procedures due to patch dehiscence and residual shunt ${ }^{(10)}$. For unbalanced complete AVSD, repair technique may include single ventricle palliation with the staged biventricular repair or primary biventricular repair ${ }^{(\mathbf{8})}$.

\section{Statistical analysis}

Statistical analysis was conducted using SPSS (Statistical Package for the Social Sciences) computer program, version 22. Categorical data were represented as numbers and frequencies. Continuous data were tested for normality by the Shapiro-Wilk test. Data were skewed and were presented as the median and interquartile range $\left(25^{\text {th }}-75^{\text {th }}\right.$ percentiles). Fishers' Exact and the Mann-Whitney U tests were performed to investigate the association between the risk factors and the incidence of in-hospital mortality and second unplanned operation. P-value less than 0.05 was considered statistically significant.

\section{RESULTS}

This study included 65 Down syndrome patients who underwent surgical repair of complete AVSD. The highest percent were females $(58.5 \%)$. Their ages at operation time ranged from 4.0 to 47.0 months with a median of 8.0 months (IQR=6.0-12.0), and the median weight at operation was $5.5(4.5-7.0) \mathrm{kg}$. The most frequent type was type A (86.2\%), followed by type C $(10.8 \%)$. Twenty- three $(35.4 \%)$ patients had associated cardiovascular anomalies, two patients $(3.1 \%)$ had TF, another 3 patients had combined $\mathrm{TF}$ and pulmonary stenosis, and Pulmonary HTN was detected in 8 patients $(12.3 \%)$.

Anatomy of $\mathrm{AV}$ valve as determined by the preoperative TEE and moderate/severe AV valve regurgitation was determined in 29 (44.6\%) patients. Preoperative catheter evaluation was done in 11 (16.9\%). Seven $(0.8 \%)$ patients underwent palliative pulmonary banding. Operative characteristics showed the use of two patches with valve annuloplasty technique for repairing in $50.8 \%$ of the patients. Further, total thymectomy was done in more than half $(52.3 \%)$ of patients. Intraoperative TEE revealed significant findings (moderate/severe valve regurgitation or stenosis with or without residual shunt) in 11 (16.9\%) patients (Table 1). 
Table (1): Demographic, preoperative, and operative characteristics of the studied patients ( $\mathrm{n}=65)$

\begin{tabular}{|c|c|c|c|}
\hline \multirow[t]{2}{*}{$\operatorname{Sex}(n, \%)$} & Female & 38 & $58.5 \%$ \\
\hline & Male & 27 & $41.5 \%$ \\
\hline \multirow[b]{2}{*}{ Age (Months) } & Range & \multicolumn{2}{|c|}{$4.0-47.0$} \\
\hline & Median (IQR) & \multicolumn{2}{|c|}{$8.0(6.0-12.0)$} \\
\hline \multirow[b]{2}{*}{ Weight (kg) } & Range & \multicolumn{2}{|c|}{$3.0-17.0$} \\
\hline & Median (IQR) & \multicolumn{2}{|c|}{$5.5(4.5-7.0)$} \\
\hline \multirow[t]{3}{*}{ Type of AVSD (n, \%) } & $\mathrm{A}$ & 56 & $86.2 \%$ \\
\hline & B & 2 & $3.1 \%$ \\
\hline & $\mathrm{C}$ & 7 & $10.8 \%$ \\
\hline \multirow[t]{6}{*}{ Associated cardiac problems (n, \%) } & PHT & 8 & $12.3 \%$ \\
\hline & Pulmonary stenosis & 3 & $4.6 \%$ \\
\hline & Pulmonary stenosis/TOF & 3 & $4.6 \%$ \\
\hline & TOF & 2 & $3.1 \%$ \\
\hline & Aortic coarctation & 1 & $1.5 \%$ \\
\hline & others & 6 & $9.2 \%$ \\
\hline \multirow[t]{2}{*}{ Anatomy of AV Valve by TEE (n, \%) } & Less than moderate AV regurgitation & 36 & $55.4 \%$ \\
\hline & $\begin{array}{l}\text { Moderate/severe AV valve } \\
\text { regurgitation }\end{array}$ & 29 & $44.6 \%$ \\
\hline Preoperative catheter evaluation $(n, \%)$ & Yes & 11 & $16.9 \%$ \\
\hline PAB $(n, \%)$ & Yes & 7 & $10.8 \%$ \\
\hline Gradient pressure around the band & Median (IQR) & \multicolumn{2}{|c|}{$80.0(7.0-122.0)$} \\
\hline Time between banding and the operation & Median (IQR) & \multicolumn{2}{|c|}{$12.0(8.0-16.0)$} \\
\hline \multirow[t]{2}{*}{ Type of patch repair $(n, \%)$} & Single patch with valve annuloplasty & 32 & 49.2 \\
\hline & Two patches \& valve annuloplasty & 33 & 50.8 \\
\hline \multirow[t]{2}{*}{ Thymectomy } & Partial & 31 & 47.7 \\
\hline & Total & 34 & 52.3 \\
\hline CPB time (Minutes) & Median (IQR) & \multicolumn{2}{|c|}{$122.0(100.0-162.0)$} \\
\hline Cross clamp time (Minutes) & Median (IQR) & \multicolumn{2}{|c|}{$89.0(68.0-120.0)$} \\
\hline \multirow[t]{2}{*}{ Intraoperative TEE } & $\begin{array}{l}\text { Significant findings (moderate/severe } \\
\text { valve regurgitation or stenosis with or } \\
\text { without residual shunt) }\end{array}$ & 11 & $16.9 \%$ \\
\hline & Others & 54 & $83.1 \%$ \\
\hline
\end{tabular}

TEE; transesophageal echocardiography, PAB; pulmonary artery banding

Table (2) showed outcomes of surgical repair of complete AVSD in the studied patients. During hospital treatment, 11 (16.9\%) stayed for longer than 10 days, $16(24.6 \%)$ were ventilated for more than $48 \mathrm{~h}$, and $30(46.2 \%)$ developed various early complications; of those, pleural effusion (chyle or not) was the most frequent (18.5\%).

Renal dysfunction with dialysis and sepsis were verified in 11 (16.9\%), while 13 (20\%) developed postoperative pulmonary HTN crisis. In-hospital mortality was documented in $3(4.6 \%)$ patients. Last follow up TEE revealed significant findings (moderate/severe mitral or tricuspid regurgitation or stenosis, with or without residual shunt) in $12(18.5 \%)$ patients, whereas $4(6.2 \%)$ needed second unplanned operation for valve/shunt correction. Heart block that needed PPM insertion was recorded in 3 (4.6\%) patients. 
Table (2): Outcomes of surgical repair of complete AVSD in the studied patients

\begin{tabular}{|c|c|c|c|}
\hline \multicolumn{2}{|c|}{ 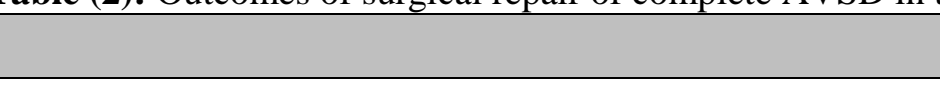 } & $\mathrm{N}=65$ & $\%$ \\
\hline \multicolumn{4}{|c|}{ Outcomes during hospital treatment } \\
\hline \multicolumn{2}{|c|}{$\begin{array}{l}\text { ICU stay longer than } 10 \text { days } \\
\text { Yes }\end{array}$} & 11 & $16.9 \%$ \\
\hline \multicolumn{2}{|c|}{ Prolonged ventilation $>48 \mathrm{~h}$} & 16 & $24.6 \%$ \\
\hline \multirow[t]{4}{*}{ Early complications } & Pleural effusion (chyle or not) & 12 & $18.5 \%$ \\
\hline & Low cardiac output & 6 & 9.25 \\
\hline & Arrythmias & 5 & $7.7 \%$ \\
\hline & Others & 7 & $10.8 \%$ \\
\hline \multicolumn{2}{|c|}{ Renal Dysfunction with dialysis } & 11 & $16.9 \%$ \\
\hline \multicolumn{2}{|c|}{ Postoperative pulmonary HTN crisis } & 13 & $20.0 \%$ \\
\hline \multicolumn{2}{|c|}{ Neurological insult } & 2 & $3.1 \%$ \\
\hline \multicolumn{2}{|c|}{ Deep sternal wound infection } & 5 & $7.7 \%$ \\
\hline \multicolumn{2}{|c|}{ Sepsis } & 11 & $16.9 \%$ \\
\hline \multicolumn{2}{|l|}{ In-hospital mortality } & 3 & $4.6 \%$ \\
\hline \multicolumn{4}{|l|}{ Long-term outcomes } \\
\hline \multirow[t]{2}{*}{ Last follow up TEE } & Significant findings ${ }^{\mathrm{a}}$ & 12 & $18.5 \%$ \\
\hline & Others & 53 & $81.5 \%$ \\
\hline \multicolumn{2}{|c|}{ Residual VSD closed by device } & 1 & $1.5 \%$ \\
\hline \multicolumn{2}{|c|}{ Heart block needed PPM insertion } & 3 & $4.6 \%$ \\
\hline \multicolumn{2}{|c|}{ Second unplanned operation } & 4 & $6.2 \%$ \\
\hline \multicolumn{2}{|c|}{ Recurrent hospital admission } & 4 & $6.2 \%$ \\
\hline
\end{tabular}

aSignificant finding: moderate/severe mitral or tricuspid regurgitation or stenosis, with or without residual shunt

Table (3) showed that in-hospital mortality was significantly associated with prolonged CPB time $(\mathrm{p}=0.008)$. The median CPB time was significantly higher in non-survivors $(220.0, \mathrm{IQR}=170.0-242.0)$ than in survivors $(121.0$, $\mathrm{IQR}=99.0-160.0$ ). As well, the development of renal dysfunction that required dialysis or sepsis during the ICU care was significantly associated with the in-hospital mortality $(\mathrm{p}=0.004)$. 
Table (3): Factors associated with In-hospital mortality

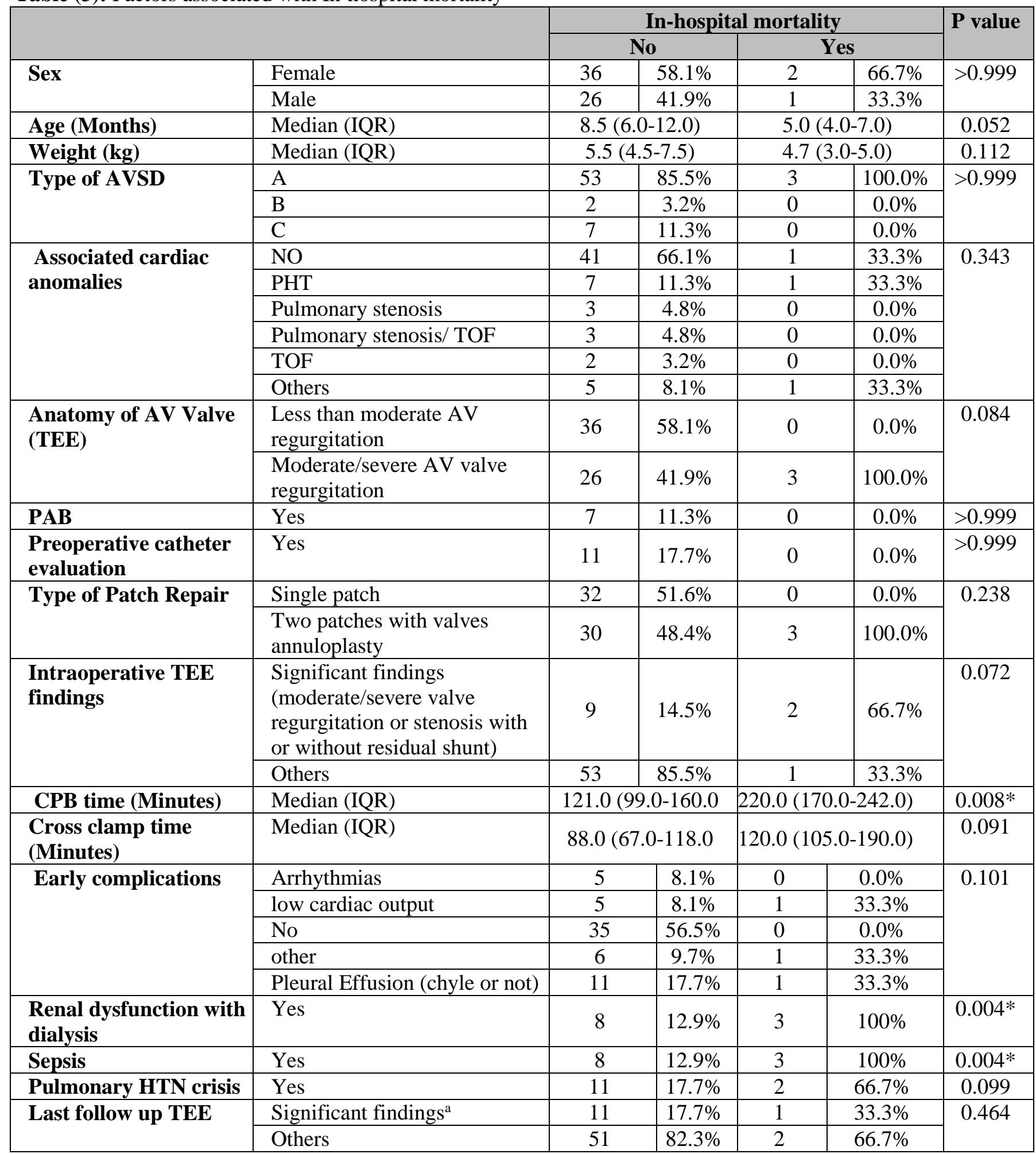

*Significant at $\mathrm{p}<0.05$ by Fisher's Exact and Mann-Whitney U tests

a'Significant finding: moderate/severe mitral or tricuspid regurgitation or stenosis, with or without residual shunt

Table (4) demonstrated a significant association between the need for second unplanned operation and type A CAVSD $(p=0.041)$. The presence of preoperative moderate/severe AV valve regurgitation as detected in the TEE $(\mathrm{p}=0.035)$, the development of pleural effusion (chyle or not) as an early postoperative complication $(\mathrm{p}=0.016)$, and the detection of significant findings (moderate/severe mitral or tricuspid regurgitation or stenosis, with or without residual shunt) in the last follow up TEE ( $\mathrm{p}=0.014$ ) were associated with need for $2^{\text {nd }}$ unplanned operation. 
Table (4): Factors associated with the need for second unplanned operation

\begin{tabular}{|c|c|c|c|c|c|c|}
\hline \multirow{2}{*}{\multicolumn{2}{|c|}{ 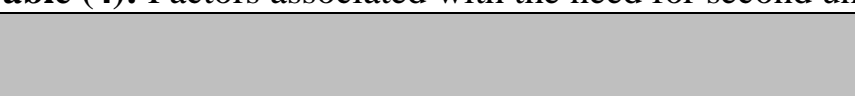 }} & \multicolumn{4}{|c|}{ Second unplanned operation } & \multirow{3}{*}{\begin{tabular}{|l} 
P value \\
0.135
\end{tabular}} \\
\hline & & \multicolumn{2}{|c|}{ No } & \multicolumn{2}{|c|}{ Yes } & \\
\hline \multirow[t]{2}{*}{ Sex } & Female & 34 & $55.7 \%$ & 4 & $100.0 \%$ & \\
\hline & Male & 27 & $44.3 \%$ & 0 & $0.0 \%$ & \\
\hline Age (Months) & Median (IQR) & \multicolumn{2}{|c|}{$8.0(6.0-12.0)$} & \multicolumn{2}{|c|}{$6.5(5.0-10.0)$} & 0.375 \\
\hline Weight (kg) & Median (IQR) & \multicolumn{2}{|c|}{$5.5(4.6-7.5)$} & \multicolumn{2}{|c|}{$5.3(4.3-6.2)$} & 0.435 \\
\hline \multirow[t]{3}{*}{ Type of AVSD } & A & 54 & $88.5 \%$ & 2 & $50.0 \%$ & \multirow[t]{3}{*}{$0.041^{*}$} \\
\hline & B & 1 & $1.6 \%$ & 1 & $25.0 \%$ & \\
\hline & $\mathrm{C}$ & 6 & $9.8 \%$ & 1 & $25.0 \%$ & \\
\hline \multirow{7}{*}{$\begin{array}{l}\text { Associated } \\
\text { cardiac } \\
\text { anomalies }\end{array}$} & Aortic C & 1 & $1.6 \%$ & 0 & $0.0 \%$ & \multirow[t]{7}{*}{0.365} \\
\hline & $\mathrm{NO}$ & 40 & $65.6 \%$ & 2 & $50.0 \%$ & \\
\hline & other & 6 & $9.8 \%$ & 0 & $0.0 \%$ & \\
\hline & PHT & 7 & $11.5 \%$ & 1 & $25.0 \%$ & \\
\hline & Pulmonary stenosis & 3 & $4.9 \%$ & 0 & $0.0 \%$ & \\
\hline & Pulmonary stenosis/ TOF & 2 & $3.3 \%$ & 1 & $25.0 \%$ & \\
\hline & TOF & 2 & $3.3 \%$ & 0 & $0.0 \%$ & \\
\hline \multirow[t]{2}{*}{$\begin{array}{l}\text { Anatomy of AV } \\
\text { Valve (TEE) }\end{array}$} & $\begin{array}{l}\text { Less than moderate AV } \\
\text { regurgitation }\end{array}$ & 36 & $59.0 \%$ & 0 & $0.0 \%$ & \multirow[t]{2}{*}{$0.035 *$} \\
\hline & $\begin{array}{l}\text { Moderate/severe AV valve } \\
\text { regurgitation }\end{array}$ & 25 & $41.0 \%$ & 4 & $100.0 \%$ & \\
\hline PAB & Yes & 6 & $9.8 \%$ & 1 & $25.0 \%$ & 0.373 \\
\hline $\begin{array}{l}\text { Preoperative } \\
\text { catheter } \\
\text { evaluation }\end{array}$ & Yes & 11 & $18.0 \%$ & 0 & $0.0 \%$ & $>0.999$ \\
\hline \multirow{2}{*}{$\begin{array}{l}\text { Type of Patch } \\
\text { Repair }\end{array}$} & Single patch & 29 & $47.5 \%$ & 3 & $75.0 \%$ & \multirow[t]{2}{*}{0.355} \\
\hline & $\begin{array}{l}\text { Two patches with valves } \\
\text { annuloplasty }\end{array}$ & 32 & $52.5 \%$ & 1 & $25.0 \%$ & \\
\hline $\begin{array}{l}\text { CPB time } \\
\text { (Minutes) }\end{array}$ & Median (IQR) & \multicolumn{2}{|c|}{$\begin{array}{c}123.0 \\
(99.0-162.0)\end{array}$} & \multicolumn{2}{|c|}{$\begin{array}{c}121.0 \\
(119.0-178.0)\end{array}$} & 0.626 \\
\hline $\begin{array}{l}\text { Cross clamp time } \\
\text { (Minutes) }\end{array}$ & Median (IQR) & \multicolumn{2}{|c|}{$\begin{array}{c}87.0 \\
(67.0-120.0)\end{array}$} & \multicolumn{2}{|c|}{$\begin{array}{c}97.5 \\
(92.5-147.0 \\
\end{array}$} & 0.293 \\
\hline \multirow{5}{*}{$\begin{array}{l}\text { Early } \\
\text { complications }\end{array}$} & Arrhythmia & 4 & $6.6 \%$ & 1 & $25.0 \%$ & \multirow[t]{5}{*}{$0.016^{*}$} \\
\hline & low cardiac output & 6 & $9.8 \%$ & 0 & $0.0 \%$ & \\
\hline & No & 35 & $57.4 \%$ & 0 & $0.0 \%$ & \\
\hline & Others & 7 & $11.5 \%$ & 0 & $0.0 \%$ & \\
\hline & Pleural effusion (chyle or not) & 9 & $14.8 \%$ & 3 & $75.0 \%$ & \\
\hline \multirow{2}{*}{$\begin{array}{l}\text { Last follow up } \\
\text { TEE }\end{array}$} & Others & 52 & $85.2 \%$ & 1 & $25.0 \%$ & \multirow[t]{2}{*}{$0.014 *$} \\
\hline & Significant findings $\mathrm{s}^{\mathrm{a}}$ & 9 & $14.8 \%$ & 3 & $75.0 \%$ & \\
\hline
\end{tabular}

*Significant at $\mathrm{p}<0.05$ by Fisher's Exact and Mann-Whitney U tests

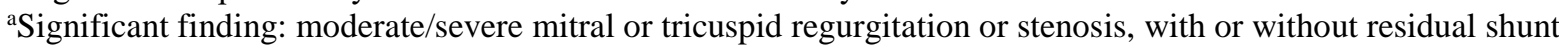

\section{DISCUSSION}

Down syndrome is accompanied with congenital cardiovascular defects in more than half of cases. The most frequently detected anomalies are CAVSD. Complete AVSD is a complex heart anomaly characterized by variable defects in both atria and ventricles, the septum and the AV valves ${ }^{(11)}$.

This study reports the experience of our institution in surgical correction of CAVSD in DS patients. Inhospital mortality was recorded in $3(4.6 \%)$ cases, while the rate of second unplanned operation mainly for correction of valves regurgitation and residual shunt was $6.2 \%$. Other outcomes included heart block that needed PPM insertion in $3(4.6 \%)$ patients and the need for hospital readmission in $4(6.2 \%)$ of patients. In-hospital mortality was significantly associated prolonged CPB time and the development of renal dysfunction that required dialysis or sepsis during the ICU care. Patients with type A CAVSD, preoperative moderate/severe AV valve regurgitation, postoperative moderate/ severe mitral or tricuspid regurgitation or stenosis, with or without residual shunt as detected in the last follow up TEE, or those who developed pleural effusion (chyle or not) during 
ICU care were at increased risk for a second unplanned operation.

In-hospital mortality was documented in $3(4.6 \%)$ patients in the present study. In-hospital mortality was significantly associated with prolonged CPB time and the development of renal dysfunction that required dialysis or sepsis during the ICU care. A comparable study included 198 children (78\% of them were DS patients) who underwent CAVSD repair over a long period from 1974 to 2000 at a single tertiary care center reported an overall early mortality, defined as death within 30 days after CAVSD repair or before hospital discharge of $10.1 \%$ that significantly decreased to $2.9 \%$ in the late surgical era from 1991

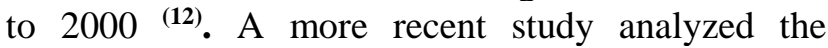
outcomes of surgical repair of CAVSD in patients younger than 2 years, of whom $70 \%$ were DS recorded hospital mortality of seven (5\%) cases. Moreover, they concluded that preoperative and postoperative AV valve regurgitation did not significantly affect patient' survival. Much higher incidence of 30-day mortality (15\%) was recorded by Al-Hay et al. ${ }^{(13)}$ who consecutively recruited 147 children underwent CAVSD repair between 1986 and 1998, 106 of them had DS.

Better understanding of the anatomy and optimization of surgical techniques have improved early clinical outcome of surgical correction of CAVSD. However, a high risk of reoperation up to $10 \%$ has been identified. Moreover, reoperations have been recognized as significant risk factor for late mortality after primary CAVSD repair ${ }^{(\mathbf{1 2})}$.

In this work, $4(6.2 \%)$ patients needed second unplanned operation. The indications were correction of mitral valve regurgitation associated with VSD in two cases, only mitral valve repair in one case, and pulmonary valvoplasty in another case. An earlier study included partial and complete AVSD primary repairs reported left atrioventricular valve regurgitation as the most common indications for reoperation that may be accompanied with right atrioventricular valve regurgitation, ventricular septal defect, or atrial septal defect ${ }^{(\mathbf{1 4})}$. Further experience in another institution reported higher incidence of reoperation (14.2\%) for moderate/severe AV valve regurgitation ${ }^{(\mathbf{1 5})}$. The reported low rate of reoperation in our study agrees with Dawary et al. (16) who reported a significantly lower reoperation rates among Down patients versus non-Down patients. They attributed this difference to the more redundant tissues in Down patients than non-Down patients did, which helps with the repair. An earlier study comprised 87 patients with CAVSD with $74 \%$ diagnosed with Down syndrome also concluded that DS is not a risk factor for reoperation ${ }^{(17)}$.

Patients with type A CAVSD, preoperative moderate/severe AV valve regurgitation, postoperative moderate/severe mitral or tricuspid regurgitation or stenosis, with or without residual shunt as detected in the last follow up TEE, or those who developed pleural effusion (chyle or not) during ICU care were at increased risk for a second unplanned operation. This is in line with Ijsselhof $\boldsymbol{e t}$ al. (18) who concluded that residual left and right atrioventricular valve regurgitation and abnormal conduction at discharge were among the subcomponents strongly associated with postdischarge reinterventions.

Complete AVSD has been classified into three types by Rastelli et $\boldsymbol{a l} .{ }^{(19)}$ according to the insertion of the chordae and the structure of the superior bridging leaflet of the common $\mathrm{AV}$ valve. In our study, type A in which the superior bridging leaflet of the common atrioventricular valve is attached to the left ventricular surface of the interventricular septum with the help of chordae showed a significant association with the risk of reoperation.

Surgical management of these complex anomalies can be achieved through different procedures that include using a single patch, double patch, or a modified single patch technique. In our study, the type of the patch whether single or double did not significantly contribute neither to mortality nor to the reoperation. This finding agrees with Dawary et al. (16), Pan et al. ${ }^{(20)}$ and Fong et al. ${ }^{(21)}$.

In the current study, heart block that needed PPM insertion was recorded in $3(4.6 \%)$ patients. Dawary et $\boldsymbol{a l}{ }^{\left({ }^{(16)}\right.}$ recorded a higher incidence of postoperative arrhythmia (14\%), of whom $11 \%$ required pacemaker insertion. As well, Günther et al. ${ }^{(22)}$ reported a comparable incidence of PPM insertion (5\%) while Crawford and Stroud ${ }^{(23)}$ reported a slightly higher incidence of $6.4 \%$.

One of the most frequent early complication in this study was pleural effusion (chyle or not) that was recorded in 12 (18.5\%) patients. Furthermore, both sepsis and renal dysfunction were determined in 11 (16.9\%) each, and they were significantly associated with in hospital mortality. A study described the outcomes of surgical repair of CAVSD in DS patients versus patients having CAVSD, but with normal karyotype. They reported higher incidence of infectious complications (21\%)in DS patients ${ }^{(9)}$. Dawary et al. (16) also reported higher frequency (23\%) of infectious complications in a mixed cohort of DS and non-DS patients. Furthermore, Günther $\boldsymbol{e t}$ al. ${ }^{(22)}$ reported death of 10 patients out of 52 who developed infectious complications following CAVSD repair.

The retrospective study design that might carry risk of low quality of data collection, besides being a single institution experience are considered limitations of this study. Further, the uncommon nature of CAVSD in comparison to other congenital 
cardiac defects, in addition to the directed objective of studying the outcomes in only Down syndrome patients contributed to the small sample size. Further the unbalanced groups of comparison regarding the number of patients in each group made the development of a risk prediction model for mortality and reoperation to be difficult.

\section{CONCLUSION}

In view of the incidence of the hospital mortality, reoperation, and other postoperative morbidities, we suggest that our outcomes are accepted for surgical repair of CAVSD in Down patients. The CPB time, the development of renal dysfunction that required dialysis or sepsis during the ICU care significantly contributed to the hospital mortality. The presence of preoperative and postoperative AV valve regurgitation and/or shunts significantly contributed to the risk of reoperation but did not show a significant relation to hospital mortality.

\section{REFERENCES}

1. Rigby M (2021): Atrioventricular Septal Defect: What Is in a Name? Journal of Cardiovascular Development and Disease, 8: 19-25.

2. Calabrò R, Limongelli G (2006): Complete atrioventricular canal. Orphanet J Rare Dis., 1: 8-13.

3. Calkoen E, Hazekamp M, Blom $\mathrm{N}$ et al. (2016): Atrioventricular septal defect: From embryonic development to long-term follow-up. Int J Cardiol., 202: 784-95.

4. Craig B (2006): Atrioventricular septal defect: from fetus to adult. Heart (British Cardiac Society), 92: 18791885.

5. German C, Nanda N (2015): Three-dimensional echocardiographic assessment of atrial septal defects. Ann Card Anaesth., 18: 69-73.

6. Bravo-Valenzuela $\mathbf{N}$, Peixoto A, Araujo Júnior $\mathbf{E}$ (2018): Prenatal diagnosis of congenital heart disease: A review of current knowledge. Indian Heart Journal, 70: 150-164.

7. Wu Y, Kuang H, Wang G et al. (2020): Surgical Management for Complete Atrioventricular Septal Defects: A Systematic Review and Meta-Analysis. Pediatr Cardiol., 41: 1445-1457.

8. Chauhan S (2018): Atrioventricular septal defects. Ann Card Anaesth., 21: 1-3.

9. Tumanyan M, Filaretova O, Chechneva $\mathrm{V}$ et al. (2015): Repair of Complete Atrioventricular Septal Defect in Infants with Down Syndrome: Outcomes and Long-Term Results. Pediatric Cardiology, 36: 71-75.

10. Vida V, Tessari C, Castaldi B et al. (2016): Early Correction of Common Atrioventricular Septal Defects:
A Single-Center 20-Year Experience. Ann Thorac Surg., 102: 2044-2051.

11. Mureşan D, Mărginean C, Zaharie G et al. (2016): Complete atrioventricular septal defect in the era of prenatal diagnosis. Med Ultrason., 18: 500-507.

12. Ginde S, Lam J, Hill G et al. (2015): Long-term outcomes after surgical repair of complete atrioventricular septal defect. J Thorac Cardiovasc Surg., 150: 369-74.

13. Al-Hay A, Macneill S, Yacoub M et al. (2003): Complete atrioventricular septal defect, Down syndrome, and surgical outcome: risk factors. The Annals of Thoracic Surgery, 75: 412-421.

14. Birim O, Van Gameren M, De Jong P et al. (2009): Outcome after reoperation for atrioventricular septal defect repair. Interact Cardiovasc Thorac Surg., 9: 8387.

15. Sivalingam S, Krishnasamy S, Afeena Al-Fahmi $\mathbf{N}$ et al. (2013): Early and midterm outcome of complete Atrioventricular Septal Defect (AVSD) in a single institution. Indian Journal of Thoracic and Cardiovascular Surgery, 29: 223-229.

16. Dawary M, Alshamdin F, Alkhalaf L et al. (2019): Outcomes of surgical repair of complete atrioventricular canal defect in patients younger than 2 years of age. Annals of Saudi Medicine, 39: 422-425.

17. Rizzoli G, Mazzucco A, Maizza F et al. (1992): Does Down syndrome affect prognosis of surgically managed atrioventricular canal defects? J Thorac Cardiovasc Surg., 104: 945-53.

18. Ijsselhof $R$, Gauvreau $K$, Nido $P$ et al. (2020): Atrioventricular Valve Function Predicts Reintervention in Complete Atrioventricular Septal Defect. World J Pediatr Congenit Heart Surg., 11: 247248.

19. Rastelli G, Kirklin J, Titus J (1966): Anatomic observations on complete form of persistent common atrioventricular canal with special reference to atrioventricular valves. Mayo Clin Proc., 41: 296-308.

20. Pan G, Song L, Zhou X et al. (2014): Complete atrioventricular septal defect: comparison of modified single-patch technique with two-patch technique in infants. J Card Surg., 29: 251-5.

21. Fong L, Betts K, Bell D et al. (2020): Complete atrioventricular septal defect repair in Australia: Results over 25 years. J Thorac Cardiovasc Surg., 159: 10141025.

22. Günther T, Mazzitelli D, Haehnel C et al. (1998): Long-term results after repair of complete atrioventricular septal defects: analysis of risk factors. Ann Thorac Surg., 65: 754-759.

23. Crawford F, Stroud M (2001): Surgical repair of complete atrioventricular septal defect. Ann Thorac Surg., 72: 1621-1628. 